\title{
Gross and histopathology lesions of abomasum of kid died of Floppy kid syndrome
}

\author{
Manju K Mathew ${ }^{1}$, S Ajithkumar ${ }^{2}$, Usha N Pillai ${ }^{3}$, G Radhika $^{4}$, P C Alex ${ }^{5}$ \\ 1,2,3,5 Dept. of Clinical Veterinary Medicine, College of Veterinary and Animal Sciences, Mannuthy, Kerala \\ Veterinary and Animal Sciences University, Kerala, India, 680651. ${ }^{4}$ Centre for Advanced Studies in Animal \\ Genetics and Breeding, College of Veterinary and Animal Sciences, Kerala Veterinary and Animal Sciences \\ University, Kerala, India
}

\begin{abstract}
D-lactic acidosis or Floppy kid syndrome (FKS) is a disease condition in kids characterized by decline in blood $\mathrm{pH}$ and elevated anion gap resulting in metabolic acidosis. A case of six day old goat kid was reported at University Goat and Sheep farm with clinical signs and laboratory findings suggestive of FKS. In spite of intense therapy the kid succumbed to death on third day of treatment. Distended abomasum with hemorrhage was seen on gross observation. Histopathology revealed abomassitis with hemorrhagic streaks.
\end{abstract}

Keywords: Abomassitis, hemorrhage, clotted milk

\section{Case Presentation}

A six day old female kid was reported at University Goat and Sheep farm, College of Veterinary and Animal Sciences, Mannuthy, Kerala Veterinary and Animal Sciences University, Kerala, India with the complaint that the kid was weak since three days. It was not suckling the dam and was unable to stand and bear weight on limbs. The kid was under treatment with the antibiotic Penicillin, 5\% dextrose and a multivitamin preparation for three days but no improvement in condition was observed.

On presentation the kid was very weak and was subjected to detailed clinical examination. Complete hematology and serum biochemical values were within normal range. Major changes noticed were on blood gas analysis. Blood $\mathrm{pH}$ was found to be 7.026. Decrease in bicarbonate level $(7.8 \mathrm{mEq} / \mathrm{L})$ and base excess of blood ($21.4 \mathrm{mmol} / \mathrm{L}$ ) and an elevated anion gap (35) was also observed. Blood gas analysis results were suggestive of severe metabolic acidosis. Total lactate level was found to be highly elevated $(21.37 \mathrm{mg} / \mathrm{dL})$ whereas L-lactate was found to be $1.58 \mathrm{mg} / \mathrm{dL}$ suggesting D-lactate elevation as the cause of metabolic acidosis in the kid. The kid was then treated with $7.5 \%$ sodium bicarbonate based on the formula (Body weight in $\mathrm{kg} \times 0.5 \times$ (-base excess) $=\mathrm{mEq}$ sodium bicarbonate), but the condition deteriorated on day two and the animal succumbed to death and was subjected to postmortem.

Abdomen was distended and on opening the abdominal cavity, abomasum was found to be highly enlarged and almost reaching pelvic cavity and occupying the whole of abdomen. On opening the abomasum was found to be filled with clotted milk (Fig 1) and to a lesser extend with gas. Abomasal mucosa was severely inflamed and was found to be hemorrhagic and edematous. Rumen was empty. Digesta was seen within duodenum but no lesion was noticed in small intestinal mucosa No lesions suggestive of any other systemic diseases were observed.

Section of abomassum obtained was subjected to histopathologcal examination after staining with haematoxylin and eosin stain. Histopathology revealed localized haemorrhage and necrosis of glandular epithelium. Submucosal damage was also observed (Fig 2,3).

\section{Discussion}

Floppy kid syndrome, a newly recognized metabolic disease is one which usually goes under-diagnosed, but it forms an important cause of neonatal mortality among goat kids. First clinical report of this disease condition was in 1987 from Canada. Disease occurs due to overconsumption of milk by the kid during the early days of their life. This leads to increased metabolism resulting in the production of D-lactate which is not produced normally in a greater quantity and is absorbed systemically resulting in D-lactate metabolic acidosis and related clinical signs. The condition if not treated at right time results in death of the affected kids. Gross changes on postmortem including distended abomasum were due to the presence of milk and milk clots and it was suggestive of pyloric functional stenosis (Klein et al., 2010). Sharp acidic odour noticed on opening the abomasum was due to the production of increased amount of lactic acid Riet-Correa et al. (2004). Petechiae observed on the abomasal mucosa might be due to the corrosive action of lactic acid (Klein et al., 2010). Cavitation and gelatinization of the cerebral hemispheres in lambs with sway-back as observed by Smith and Sherman (2009) was not noticed in the present case. 


\section{Acknowledgement}

Authors are thankful to the staff of University Goat and Sheep farm and Department of Veterinary Pathology, College of Veterinary and Animal Sciences, Mannuthy, Kerala Veterinary and Animal Sciences University, India for all the helps rendered.

\section{References}

[1]. Klein, C., Bostedt, H. and Wehrend, A. 2010. D-lactate elevation as a cause of metabolic acidosis in newborn goat kids. The potential role of milk ingestion as etiopathogenetic factor. Tierärztl Prax. 38: 371-376

[2]. Riet-Correa, F., Tabosa, I.M., de Vasconcelos, J.S. and Medeiros, J.M. 2004. Goat Mole Syndrome ("Floppy Kid") - floppy kid syndrome. Pesq. Vet. Bras. 24: 111-113

[3]. Smith, M.C. and Sherman, D.M. 2009. Nutrition and metabolic diseases. Goat medicine. John Wiley and sons, Inc, publication, USA, 871p

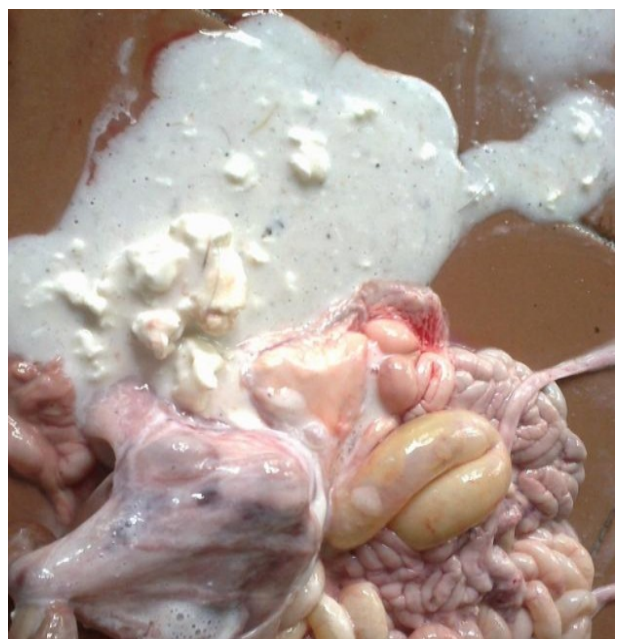

Fig 1 Abomassum on opening filled with clotted milk

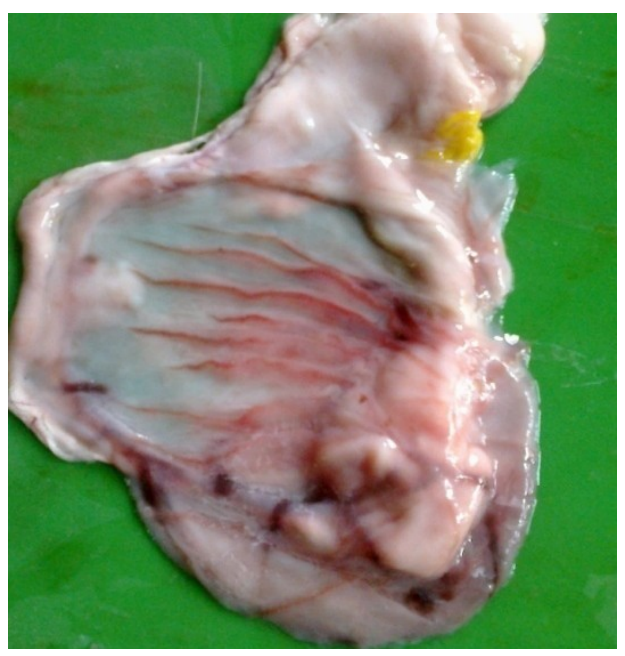

Fig3

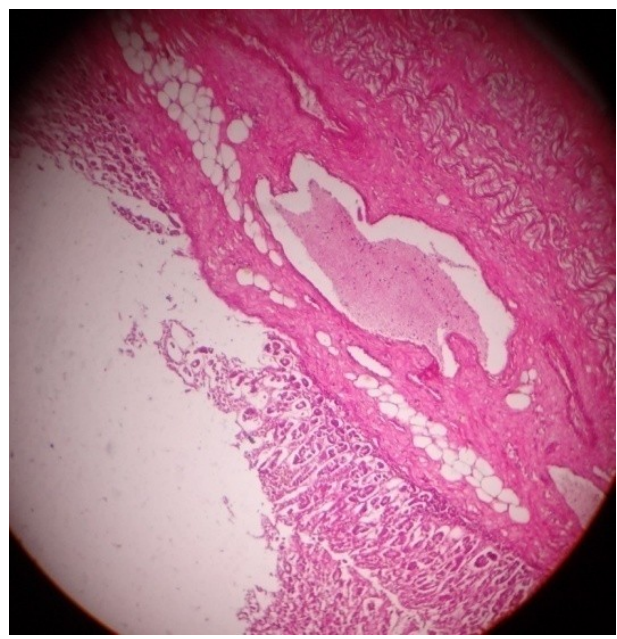

Fig 4

Odematous and hemorrhagic abomassum 